Article

\title{
In Vitro Ciliotoxicity and Cytotoxicity Testing of Repeated Chronic Exposure to Topical Nasal Formulations for Safety Studies
}

\author{
Larisa Tratnjek ${ }^{1}\left(\mathbb{D}\right.$, Nadica Sibinovska ${ }^{2}$, Katja Kristan ${ }^{3,4, *}$ and Mateja Erdani Kreft ${ }^{1, *(D)}$ \\ 1 Institute of Cell Biology, Faculty of Medicine, University of Ljubljana, Vrazov trg 2, 1000 Ljubljana, Slovenia; \\ larisa.tratnjek@mf.uni-lj.si \\ 2 Chair of Biopharmaceutics and Pharmacokinetics, Faculty of Pharmacy, University of Ljubljana, \\ Aškerčeva cesta 7, 1000 Ljubljana, Slovenia; sibinovskanadica@gmail.com \\ 3 Lek Pharmaceuticals, d.d., Sandoz Development Center Slovenia, Verovškova 57, 1526 Ljubljana, Slovenia \\ 4 Institute of Biochemistry, Faculty of Medicine, University of Ljubljana, Vrazov trg 2, 1000 Ljubljana, Slovenia \\ * Correspondence: katja.kristan@sandoz.com (K.K.); mateja.erdani@mf.uni-lj.si (M.E.K.)
}

\section{check for} updates

Citation: Tratnjek, L.; Sibinovska, N.; Kristan, K.; Kreft, M.E. In Vitro Ciliotoxicity and Cytotoxicity Testing of Repeated Chronic Exposure to Topical Nasal Formulations for Safety Studies. Pharmaceutics 2021, 13, 1750. https://doi.org/10.3390/ pharmaceutics 13111750

\section{Academic Editors:}

Tomoyuki Furubayashi and Daisuke Inoue

Received: 26 August 2021

Accepted: 15 October 2021

Published: 20 October 2021

Publisher's Note: MDPI stays neutral with regard to jurisdictional claims in published maps and institutional affiliations.

Copyright: (c) 2021 by the authors. Licensee MDPI, Basel, Switzerland. This article is an open access article distributed under the terms and conditions of the Creative Commons Attribution (CC BY) license (https:/ / creativecommons.org/licenses/by/ $4.0 /)$.

\begin{abstract}
Certain active drugs and excipients of nasal formulations may impair ciliary function and mucociliary clearance. The ciliary beat frequency $(\mathrm{CBF})$ is a key parameter for determining mucociliary clearance rate, and in vitro assessments of CBF have proven to be accurate and reproducible. Since topical nasal formulations are applied with repeated doses, it is essential to elucidate their chronic, as opposed to acute, effect on mucociliary clearance and nasal mucosa. The aim of this study was to assess for the first time the ciliotoxicity and cytotoxicity of nasal sprays intended for chronic treatment (with repeated doses) using a previously designed set-up for CBF measurements. For 2 weeks, the 3D nasal MucilAir ${ }^{\mathrm{TM}}$ in vitro models were treated daily with undiluted or clinically relevant doses of mometasone nasal spray, placebo nasal spray, culture medium, or they were untreated. We demonstrated a dose-dependent and time-dependent (cumulative) effect of the nasal sprays on ciliary activity and cytotoxicity using CBF measurements and ultrastructural analysis, respectively. Our results indicate that repeated administration of clinically relevant doses of mometasone nasal spray is safe for in vivo use, which is in good agreement with a previous clinical study. Overall, our study suggests that such in vitro assays have great potential for topical nasal drug screening.
\end{abstract}

Keywords: nasal drug formulations; mucociliary clearance; ciliary beat frequency; nasal in vitro model; repeated exposure; ciliotoxicity; cytotoxicity; ultrastructural analysis; drug safety

\section{Introduction}

Intranasal decongestants, corticosteroids, antihistamines, and anticholinergics are commonly used topical nasal drugs that are often suggested as first-line therapy for nasal conditions such as congestion, rhinitis, sinusitis, and related allergic or chronic nasal conditions [1-6]. They are administered as lavages, drops, squirt systems, or sprays and are used as repeated-dose treatments for nasal diseases [2,7]. In addition to active drugs, various formulation excipients, such as preservatives and absorption enhancers, are present in topical nasal formulations. However, it is essential that active drugs and excipients, individually or in combination, do not interfere with the integrity of the nasal defence mechanism. The main purpose of nasal mucosa is to form a physical barrier that protects the human body from inhaled foreign particles [8], and not to serve as an administration route for drug formulations. The mechanical barrier against pathogen invasion consists of intracellular junctions, mucus, and mucociliary clearance, which depends on the ciliary activity and mucus production of ciliated and goblet cells, respectively [8].

The ciliary beat frequency $(\mathrm{CBF})$ is the main parameter that determines the rate of mucociliary clearance [9]. The current clinical method for measuring CBF uses phase-contrast 
microscopy and a tissue biopsy obtained by brushing the nasal cavity [10-13]. CBF measurements have been used as an index of ciliary function to assess the ciliotoxicity of nasal drugs in vitro, which has proven to be an accurate and reproducible technique [3,14-20]. Standardised human in vitro models [21-24] are easier to use for routine testing and screening than in vivo or ex vivo models, which have the added disadvantage of interspecies differences $[25,26]$. Furthermore, clinical trials are not suitable for screening a large number of formulation candidates because they are time-consuming, expensive, and often stressful for patients [27]. Even though in vitro experiments cannot accurately mimic in vivo conditions due to a less pronounced or absent protective mucus layer, in vitro CBF analysis should become routine in the development of nasal drug formulations [14,18,28-30].

For example, benzalkonium chloride (BKC), which is used as a preservative in nasal sprays, has shown ciliotoxicity in many in vitro studies $[15,16,28,31-35]$. Its potentially toxic effect has also been demonstrated by several in vivo studies [36-40], and thus caution is advised when using BKC [41,42]. Nevertheless, the toxic effect of BKC can be neutralised in vitro by dexpanthenol, which is a protective and nurturing compound of nasal sprays $[28,43]$. Therefore, the excipients and active drugs of topical nasal formulations should be tested in vitro for ciliotoxicity and cytotoxicity individually and as combined in the final nasal formulation. Importantly, as topical nasal formulations are used as repeated doses, the cumulative effect versus the effect of single-dose exposure of the nasal formulation should also be tested. For example, repeated 5-day exposure to BKC in vitro resulted in cumulative and irreversible immediate ciliostasis, whereas this was not the case for other tested preservatives (phenylethyl alcohol) [35]. This indicates that the in vitro method can be used to screen for non-ciliotoxic excipients.

In the present study, we examined the ciliotoxicity and cytotoxicity of repeated doses of the nasal spray formulation. We have previously shown that the combination of the standardised ready-to-use 3D nasal MucilAir ${ }^{\mathrm{TM}}$ in vitro model and high-speed phase-contrast microscopy is simple and efficient for single-dose ciliotoxicity studies. A subsequent ultrastructural analysis was performed after the treatment to investigate the cytotoxicity of the nasal spray formulation [34]. The aim of this study was to evaluate the cumulative cilio- and cytotoxicity of repeated doses of nasal spray formulations using a previously developed set-up. During a period of 2 weeks, the nasal MucilAir ${ }^{\mathrm{TM}}$ in vitro models were treated daily with undiluted and clinically relevant doses of mometasone nasal spray, placebo nasal spray (without the active drug), and culture medium. Ciliotoxicity was determined with CBF measurements before treatment and then every other day (except on weekends). Cytotoxicity was investigated with the lactate dehydrogenase (LDH) assay every other day (except on weekends) and ultrastructural analysis at the end of the experiment (day 15) (Figure 1). Our results reveal the dose-dependent and time-dependent (cumulative) ciliotoxic and cytotoxic effects of the nasal sprays. The combination of the nasal MucilAir ${ }^{\mathrm{TM}}$ in vitro model and high-speed phase-contrast microscopy shows great potential as an in vitro method for the screening and testing of long-term ciliotoxicity and cytotoxicity after repeated doses in the growing field of potential nasal therapies. This could significantly contribute to the three Rs principle of refining, reducing, and replacing the use of laboratory animals. 

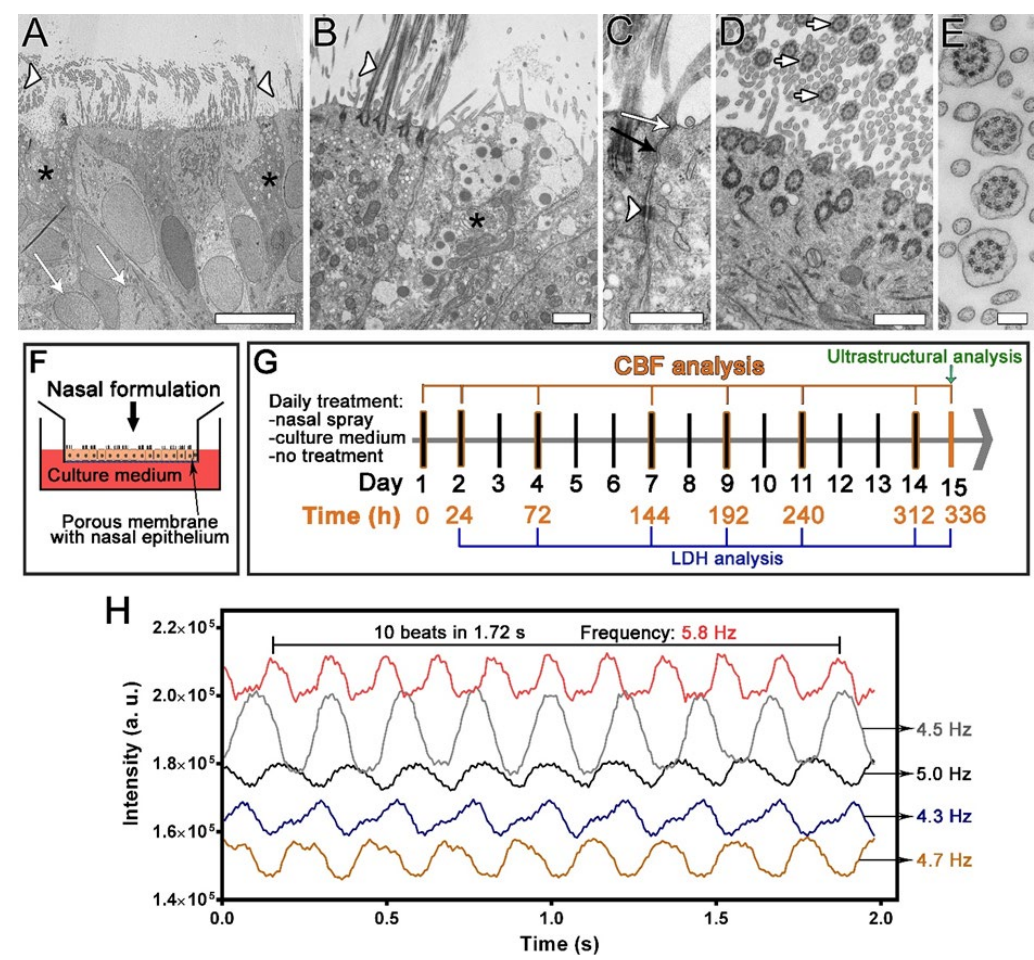

Figure 1. The design of the repeated exposure study utilising the nasal MucilAir ${ }^{\mathrm{TM}}$ in vitro models and high-speed digital phase-contrast microscopy imaging. (A-E) Ultrastructural properties of the nasal in vitro model. (A,B) A pseudostratified epithelium with ciliated cells (A,B, white arrowheads indicate cilia), goblet cells (A,B, black asterisks), and basal cells ( $\mathbf{A}$, white arrows) is formed. (C) Adjacent cells are firmly connected by tight junctions (white arrow), adherent junctions (black arrow), and desmosomes (white arrowhead). (D,E) Characteristic axonemal structure with $9 \times 2+2$ arrangement of microtubules is viewed in cross section of the cilia (white arrows, D,E). Bars, $10 \mu \mathrm{m}$ (A), $1 \mu \mathrm{m}$ (B), $600 \mathrm{~nm}$ (C,D), $200 \mathrm{~nm}$ (E). (F) Nasal spray or culture medium was applied to the apical surface of the nasal in vitro models for $30 \mathrm{~min}$ daily and then washed off. (G) Cells were treated daily for 14 days, and the ciliary beat frequency $(\mathrm{CBF})$ was analysed before the treatments $(0 \mathrm{~h})$ and at the indicated times. Ultrastructural analysis was performed at the end of the study. Lactate dehydrogenase (LDH) cytotoxicity assays were performed at the indicated times. (H) CBF measurements were performed by analysing the time-dependent greyscale intensity variations resulting from the repetitive cilia beating. Examples of the greyscale waveforms are shown, depicting the change in the grey intensity of the image at 5 different ROI as a function of time. The beat period was determined as the number of beats per unit time, from which the CBF was calculated (one divided by the beat period) and expressed in $\mathrm{Hz}$.

\section{Materials and Methods}

\subsection{Air-Liquid Culturing of the 3D Human Nasal MucilAir ${ }^{\mathrm{TM}}$ Epithelium}

The nasal MucilAir ${ }^{\mathrm{TM}}$ in vitro models were purchased from Epithelix (Geneva, Switzerland) and originated form a mixture of human nasal epithelial cells collected from 14 healthy donors (cell type hAEC/Nasal, Product Code: EP02, Lot Number: MP0008). Each reconstituted ready-to-use 3D epithelium consists of approximately 500,000 cells. According to the manufacturer's instructions, the nasal in vitro models were grown on 24-well, $6.5 \mathrm{~mm}$ diameter Transwell inserts with $0.4 \mu \mathrm{m}$ porous membranes (Corning Incorporated, Tewksbury, MA, USA, Cat\# 3470). Airway epithelial cell differentiation was triggered on day 6 by removing culture medium from the apical chamber, i.e., air-liquid interface culturing. The nasal MucilAir ${ }^{\mathrm{TM}}$ in vitro models were 2 months old upon arrival. They were cultured in a humidified incubator $\left(37^{\circ} \mathrm{C}, 5 \% \mathrm{CO}_{2}\right)$ in a proprietary and defined airway culture medium (MucilAir ${ }^{\mathrm{TM}}$ culture medium) at the air-liquid interface with culture medium present in the basal chamber only. The medium in the basal chamber $(600-700 \mu \mathrm{L})$ was replaced 
every $48 \mathrm{~h}$ (except on weekends), and apical wash was performed (with $200 \mu \mathrm{L}$ of culture medium) to remove the accumulated mucus. The morphology and ciliary activity of the nasal MucilAir ${ }^{\mathrm{TM}}$ in vitro models were observed daily under a phase-contrast microscope (DM-IL Leica, Vienna, Austria; objective C Plan 10×/0.22 Ph1 and L40×/0.50 Ph2).

\subsection{Transmission Electron Microscopy (TEM)}

The nasal in vitro models were prepared for TEM as described previously [34]. Fixation of the samples was performed for $3 \mathrm{~h}$ at $4{ }^{\circ} \mathrm{C}$ with $3 \%(w / v)$ formaldehyde (SigmaAldrich, Taufkirchen, Germany) and 3\% $(v / v)$ glutaraldehyde (Serva, Heidelberg, Germany) in $0.1 \mathrm{M}$ cacodylate buffer ( $\mathrm{pH}$ 7.4). Afterwards, the samples were rinsed overnight at $4{ }^{\circ} \mathrm{C}$ in $0.1 \mathrm{M}$ cacodylate buffer. Next, the samples were post-fixed for $1 \mathrm{~h}$ at $4{ }^{\circ} \mathrm{C}$ in $2 \%(w / v)$ osmium tetroxide (Serva). After short rinse in distilled water, the samples were incubated for $1 \mathrm{~h}$ at room temperature (RT) in 2\% uranyl acetate (Merck, Darmstadt, Germany) and rinsed again in distilled water. The samples were then dehydrated in a graded series of ethanol baths and embedded in Epon (Serva) by infiltration. Epon polymerisation was performed over the next 5 days with gradual temperature increases $\left(35^{\circ} \mathrm{C}, 45^{\circ} \mathrm{C}, 60{ }^{\circ} \mathrm{C}\right.$, $70{ }^{\circ} \mathrm{C}$, and $80^{\circ} \mathrm{C}$ ) every $24 \mathrm{~h}$. Ultrathin sections (60 nm thick) were then prepared with an ultramicrotome (Leica Microsystems, EM UC6, Vienna, Austria), contrasted with uranyl acetate and lead citrate, and examined at an operation voltage of $80 \mathrm{kV}$ with a transmission electron microscope (CM100, Philips, Eindhoven, The Netherlands) equipped with a CCD camera (AMT, Danvers, MA, USA).

\subsection{Scanning Electron Microscopy (SEM)}

The nasal in vitro models were prepared for SEM as described previously [34]. Fixation of the samples was performed for $2 \mathrm{~h}$ at $4{ }^{\circ} \mathrm{C}$ with $2 \%$ formaldehyde $(w / v)$ and $2 \%$ glutaraldehyde $(v / v)$ in $0.2 \mathrm{M}$ cacodylate buffer ( $\mathrm{pH} 7.4)$ (the fixative was added to both the apical and basal chambers). After fixation, samples were rinsed overnight at $4{ }^{\circ} \mathrm{C}$ in $0.2 \mathrm{M}$ cacodylate buffer and then post-fixed for $1 \mathrm{~h}$ at $4{ }^{\circ} \mathrm{C}$ in $1 \%(w / v)$ osmium tetroxide. Next, samples at RT were dehydrated in increasing concentrations of ethanol, acetone, and hexamethyldisilazane and then dried. Finally, the samples were sputter-coated with gold and examined at $30 \mathrm{kV}$ with a Tescan Vega3 scanning electron microscope (Brno, Czech Republic).

\subsection{Treatment with Nasal Formulations}

The nasal MucilAir ${ }^{\mathrm{TM}}$ in vitro models were incubated daily for $30 \mathrm{~min}$ with the (1) nasal spray Mommox ${ }^{\circledR} /$ Mometasone Sandoz $^{\circledR}$ (Lek Pharmaceuticals, Ljubljana, Slovenia), containing the active pharmaceutical ingredient mometasone furoate (50 mcg/actuation spray) and the excipients BKC, carboxymethylcellulose sodium, microcrystalline cellulose, sodium citrate, glycerine, citric acid, polysorbate 80, and water for injection; (2) placebo nasal spray without active pharmaceutical ingredients but containing all the above-mentioned excipients; or (3) MucilAir ${ }^{\mathrm{TM}}$ culture medium. Intact control cultures were maintained at the air-liquid interface and were not treated with nasal spray or culture medium. For treatment, nasal sprays and culture medium were added to the apical chamber of the nasal in vitro models. Nasal spray application was performed as described previously [34]. The nasal spray pump bottle was shaken, and then the suspension was first applied by spraying into a centrifuge tube and then pipetted into the apical chamber (20 or $40 \mu \mathrm{L})$. For the 10-fold diluted concentration, the Mommox ${ }^{\circledR}$ nasal spray and placebo nasal spray were diluted appropriately with culture medium supplemented with $20 \mathrm{mM}$ HEPES. For culture medium-treated nasal in vitro models, MucilAir ${ }^{\mathrm{TM}}$ culture medium was pipetted into the apical chamber $(20$ or $40 \mu \mathrm{L})$. The culture medium in the basal chamber $(600 \mu \mathrm{L})$ was changed with fresh $600 \mu \mathrm{L}$ of culture medium (on days when CBF was not measured) or HEPES-supplemented $(20 \mathrm{mM})$ culture medium (on days when CBF was measured) in all cultures, i.e., Mommox ${ }^{\circledR}$-treated, placebo-treated, and control cultures (culture mediumtreated and intact). The nasal MucilAir ${ }^{\mathrm{TM}}$ in vitro models were exposed daily (every $24 \mathrm{~h}$ ) 
for 14 consecutive days to undiluted or diluted nasal sprays and culture medium for $30 \mathrm{~min}$ at $37^{\circ} \mathrm{C}$ in a humidified incubator $\left(5 \% \mathrm{CO}_{2}\right)$. The nasal sprays were then rinsed off by apical washing of the nasal in vitro models with MucilAir ${ }^{\mathrm{TM}}$ culture medium to simulate the removal of nasal sprays from the nasal epithelium by mucociliary clearance in vivo. The culture medium in the basal chamber was changed with fresh medium. The CBF was analysed (as described in Section 2.5) before and $24 \mathrm{~h}$ after treatment and then every other day except on weekends (see the scheme of the experiment in Figure 1F-G). During the experiments, the information regarding the used nasal sprays was blinded, i.e., the operator did not know whether mometasone or placebo nasal spray was used.

\subsection{CBF Measurement}

CBF was determined as described previously [34]. The specific set-up was composed of inverted Leica DM-IL microscope with a phase-contrast objective C Plan 10×/0.22 Ph1, a high-speed recording camera (Basler acA11300-200 $\mu \mathrm{m}$, Ahrensburg, Germany), the computer connected to a USB3 port and the software Basler (Video recording software V1.1., Ahrensburg, Germany). The nasal in vitro models were first adjusted to RT for $20 \mathrm{~min}$. Recordings of ciliary beating were made in five randomly selected fields of view in each nasal in vitro model. for 1.75-2.5 s with the following settings: exposure time $6.5 \mathrm{ms,}$ video recording rate 143 frames/s. The size of the captured images was $1280 \times 1024$ pixels. The image sequences were then imported into ImageJ2 [44] using Fiji distribution [45]. On each sequence of frame-by-frame images five square (10-12 pixels) regions of interest (ROI) of different ciliated areas of the nasal in vitro models were selected with high signal-tonoise ratio of the greyscale data obtained from the ROI. Within the ROI of each frame, mean greyscale values were analysed. Areas without apparent cilia beating were not included in the analysis. The total number of analysed ROIs per nasal in vitro model was $25(n=25)$. Data were exported to Microsoft Excel (Microsoft, Redmond, WA, USA) in which mean intensity versus time was plotted (Figure $1 \mathrm{H}$ ). The variation in greyscale intensity in each ROI throughout the sequence of frame-by-frame images resulted from the repeated beating of the cilia. The beat period was determined as the number of beats (complete cycles) per unit of time (Figure 1H), from which the CBF was calculated (one divided by the beat period) and expressed in Hertz (Hz). CBF was measured on 1-2 independent nasal in vitro models, each containing cells from 14 donors, i.e., 14 biological replicates. Results are presented as the relative $\mathrm{CBF}$ values, i.e., percentages of the corresponding baseline CBF values measured on day 1 before the treatments (time point $0 \mathrm{~h}$ ). Measured absolute CBF values ranged between 3 and $8 \mathrm{~Hz}$.

\subsection{LDH Cytotoxicity Assay}

The cytotoxicity of the diluted mometasone nasal spray was investigated by performing an LDH cytotoxicity assay (CyQUANT LDH Cytotoxicity Assay Kit, Invitrogen, Thermo Fisher Scientific, Waltham, MA, USA). Inserts with nasal in vitro models were exposed to 10-fold diluted mometasone or placebo spray or culture medium, as described in Section 2.4. The withdrawn samples ( $300 \mu \mathrm{L}$ of culture medium from the basal chamber) on days $2,4,7,9,11,14$, and 15 were stored at $-20{ }^{\circ} \mathrm{C}$ until further analysis (see the scheme of the experiment in Figure 1F-G). The LDH cytotoxicity assay was performed according to the manufacturer's instructions, with minor modifications. Briefly, to obtain samples of maximum and spontaneous LDH activity, the RPMI 2650 cell suspension $\left(\approx 8.3 \times 10^{5}\right.$ cells $\left./ \mathrm{mL}\right)$ was treated for 45 min with $10 \times$ Lysis Buffer or sterile ultrapure water, respectively (the samples were prepared in duplicate). After 45 min of incubation at $37^{\circ} \mathrm{C}$, the treated cells were centrifuged at RT and $3000 \mathrm{rpm}$ for $5 \mathrm{~min}$. Next, $50 \mu \mathrm{L}$ of the supernatant samples (culture medium from the basal chamber of nasal in vitro models treated with mometasone nasal spray, placebo, or culture media or intact nasal in vitro models; spontaneous and maximum LDH activity) were transferred to a 96-well flat-bottom plate in duplicate wells, and $50 \mu \mathrm{L}$ of reaction mixture was added to each sample well. After $30 \mathrm{~min}$ of incubation at RT and protected from light, $50 \mu \mathrm{L}$ of Stop 
solution was added to each well, and the absorbance was measured at $490 \mathrm{~nm}$ and $680 \mathrm{~nm}$. The LDH activity was determined by subtracting the absorbance value at $680 \mathrm{~nm}$ (background) from the absorbance at $490 \mathrm{~nm}$ before calculating the \% cytotoxicity according to the following equation:

$$
\% \text { cytotoxicity }=\frac{\text { Compound }- \text { treated LDH activity }- \text { Spontaneous LDH activity }}{\text { Maximum LDH activity }- \text { Spontaneous LDH activity }} \times 100
$$

\subsection{Data Presentation and Statistical Analysis}

Data were analysed using the GraphPad Prism software (GraphPad, La Jolla, San Diego, CA, USA). Differences between experimental groups were tested for significance using two-way ANOVA with the Tukey's multiple comparisons test. Differences were considered statistically significant if $p \leq 0.05$. All data presented in the graphs are expressed as mean \pm S.E.

\section{Results}

\subsection{CBF Measurements in the Nasal MucilAir ${ }^{\mathrm{TM}}$ In Vitro Model}

The nasal MucilAir ${ }^{\mathrm{TM}}$ in vitro models were repeatedly treated to evaluate the chronic ciliotoxicity and cytotoxicity of nasal formulations (Figure 1). Human nasal MucilAir ${ }^{\mathrm{TM}}$ cells at the air-liquid interface formed fully differentiated pseudostratified epithelia with three cell types: ciliated, goblet, and basal cells (Figure 1A-E). Ciliated and mucus-secreting goblet cells were homogeneously distributed in the nasal in vitro models. In addition, the nasal in vitro models displayed several other in vivo characteristics such as stratification, mucus production, tight junctions (Figure 1A-E), and ciliary activity, which was analysed by recording ciliary movement with a high-speed digital camera (Supplementary Video S1). The mean (baseline) CBF of the nasal in vitro models was $4.6 \pm 0.9 \mathrm{~Hz}$ (mean \pm standard deviation, $n=18$ cultures).

\subsection{Repeated Exposure to Undiluted Nasal Sprays}

To investigate the effect of repeated exposure to mometasone nasal spray on CBF, the nasal MucilAir ${ }^{\mathrm{TM}}$ in vitro models were initially treated daily with undiluted mometasone and placebo (without the active drug) nasal spray. We have previously shown that single-exposure treatment with undiluted mometasone nasal spray for $3 \mathrm{~h}$ increases ciliary activity of nasal MucilAir ${ }^{\mathrm{TM}}$ in vitro models by $25.7 \pm 2.5 \%$ compared to culture medium-treated controls. However, $24 \mathrm{~h}$ after shorter $(30 \mathrm{~min})$ undiluted mometasone treatment, ciliary activity decreases by $18.5 \pm 9.6 \%$ compared to culture medium-treated controls [34]. In this study, repeated exposure of the nasal in vitro models to undiluted mometasone nasal spray revealed a cumulative time-dependent effect on CBF. CBF was reduced to $80.2 \% \pm 5.9 \%$ and $81.4 \% \pm 6.4 \%$ of the baseline CBF (i.e., baseline control condition, which was measured before the treatment at time point $0 \mathrm{~h}$ ) on days 2 and 4 , respectively (Figure 2). After 1 week of daily treatments with undiluted mometasone, the CBF decreased to $65.1 \% \pm 7.4 \%$ of the baseline CBF (day 7, Figure 2, Supplementary Videos S2 and S3) and was significantly lower from the culture medium-treated and intact nasal in vitro models (Figure 2, Supplementary Table S1). Ciliostasis was observed on day 9 (Supplementary Video S4). In addition, most cells detached from the growth surface. Namely, cell shedding started on day 7 in the periphery of the cultures and progressed to nearly total cell loss by day 11 (Figure 3, Supplementary Figure S1). On day 11, the experiment was terminated (CBF analysis was performed on days 1-7, Figure 2).

Similarly, daily treatment with placebo nasal spray inhibited the ciliary activity of the nasal in vitro models. A significant CBF reduction was observed on day 7 (to $69.7 \% \pm 4.7 \%$ of the baseline CBF, Figure 2, Supplementary Videos S5 and S6), which was significantly lower than the CBF of the culture medium-treated and intact nasal in vitro models (Figure 2, Supplementary Table S1). On day 11, ciliostasis and almost complete cell detachment were observed (Figure 3, Supplementary Figure S1, Supplementary Video S7). As in the mometasone nasal spray-treated cultures, cell shedding started at the periphery on day 
7 and progressed to nearly total cell loss by day 11 in placebo-treated cultures (Figure 3, Supplementary Figure S1).

Conversely, daily treatment with culture medium did not affect the ciliary activity of the nasal in vitro models (Figure 2, Supplementary Table S1, Supplementary Videos S8 and S9). No cell detachment was observed (Figure 3, Supplementary Figure S1). The CBF values even increased with time in the intact nasal in vitro models (Figure 2, Supplementary Table S1, Supplementary Videos S10 and S11).

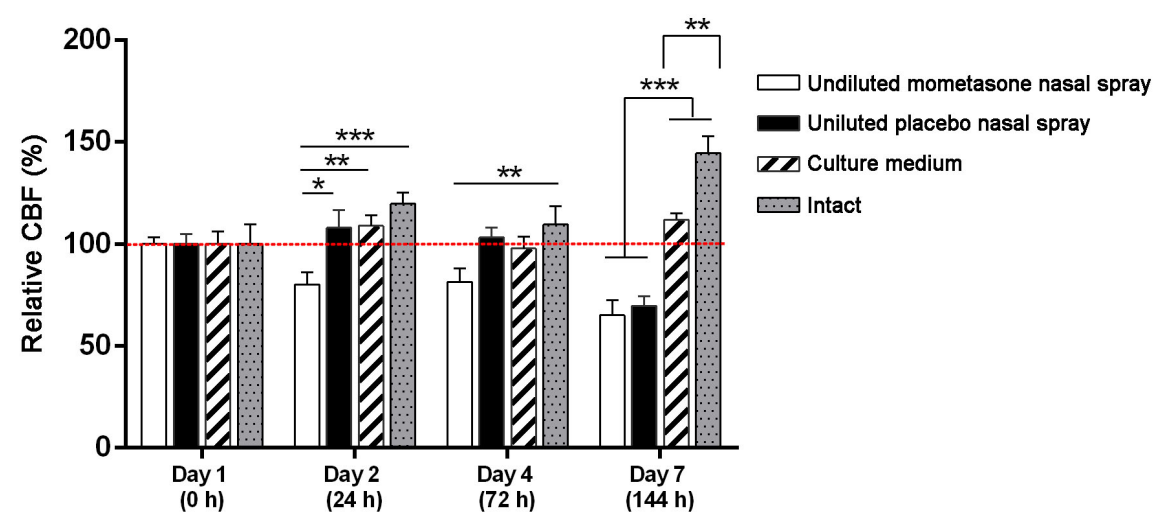

Figure 2. The effect of repeated doses of undiluted mometasone and placebo nasal spray, culture medium or intact condition on the ciliary beat frequency (CBF) of the nasal MucilAir ${ }^{\mathrm{TM}}$ in vitro model. Results are presented as relative $\mathrm{CBF}$, expressed as a percentage of the CBF values relative to the corresponding baseline control condition for each tested group measured before treatments. The red dotted line represents $100 \%$ (the value of the control condition before treatment). Each bar represents the average relative $\mathrm{CBF} \pm$ S.E. Mometasone nasal spray inhibits $\mathrm{CBF}$ as early as $24 \mathrm{~h}$ after the first treatment, and this inhibition persist on days 4 and 7. Placebo nasal spray significantly inhibits CBF on day 7 of continuous treatment. $n=25$; two-way ANOVA with Tukey's multiple comparisons test; ${ }^{*} p<0.05,{ }^{* *} p<0.01,{ }^{* * *} p<0.0001$.
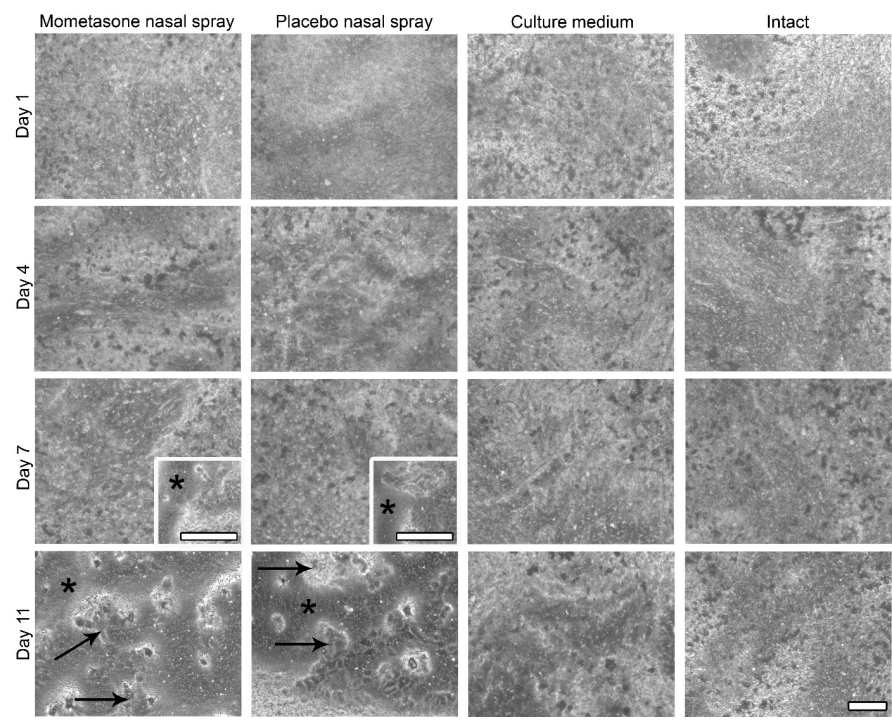

Figure 3. Morphological assessment of the nasal MucilAir ${ }^{\mathrm{TM}}$ in vitro models treated repeatedly with undiluted mometasone or placebo nasal spray, culture medium treated or untreated using phasecontrast microscopy. The apical surface view is shown. Cell shedding is observed in the undiluted mometasone- and placebo-treated nasal MucilAir ${ }^{\mathrm{TM}}$ in vitro models, which starts at the periphery on day 7 (inserts). Cell detachment is almost complete on day 11. Asterisks indicate areas without cells; arrows indicate the remaining cells on the growth surface. Scale bars, $100 \mu \mathrm{m}$. 


\subsection{Repeated Exposure to Clinically Relevant Dilutions of Nasal Sprays}

Since nasal formulations administered into the nostrils are diluted by mucus under physiological conditions, we tested the effect of repetitive exposure with 10-fold diluted concentrations of the nasal sprays. The dilution factor of 10 has been described as clinically relevant $[35,38,46]$. Mometasone nasal spray did not impair ciliary activity; no statistical differences compared to the intact nasal MucilAir ${ }^{\mathrm{TM}}$ in vitro models were found (Figure 4, Supplementary Table S2, Supplementary Videos S12-S14). Similarly, placebo nasal spray did not impair CBF compared to the intact nasal in vitro models (Figure 4, Supplementary Table S2, Supplementary Videos S15-S17). On day 2, the CBF values in the placebo-treated cultures even increased compared to the intact nasal in vitro models (Figure 4, Supplementary Table S2). Conversely, daily treatment with culture medium increased CBF in the second week of treatments. Namely, CBF increased with time compared to the baseline CBF (time point $0 \mathrm{~h}$ ). On days 9 and 14, the CBF increased compared to the mometasone-treated and intact nasal in vitro models (Figure 4, Supplementary Table S2, Supplementary Videos S18-S20 (culture medium-treated) and Supplementary Videos S21-S23 (intact nasal in vitro models)). On day 15, the mometasonetreated nasal in vitro models exhibited higher CBF values than those of the placebo- and culture medium-treated nasal in vitro models (Figure 4, Supplementary Table S2).

However, we observed cell shedding at the periphery of the mometasone- and placebo-treated nasal in vitro models on days 14 and 15. Conversely, cell shedding was not observed in the culture medium-treated and intact nasal in vitro models (Figure 5, Supplementary Figure S2).

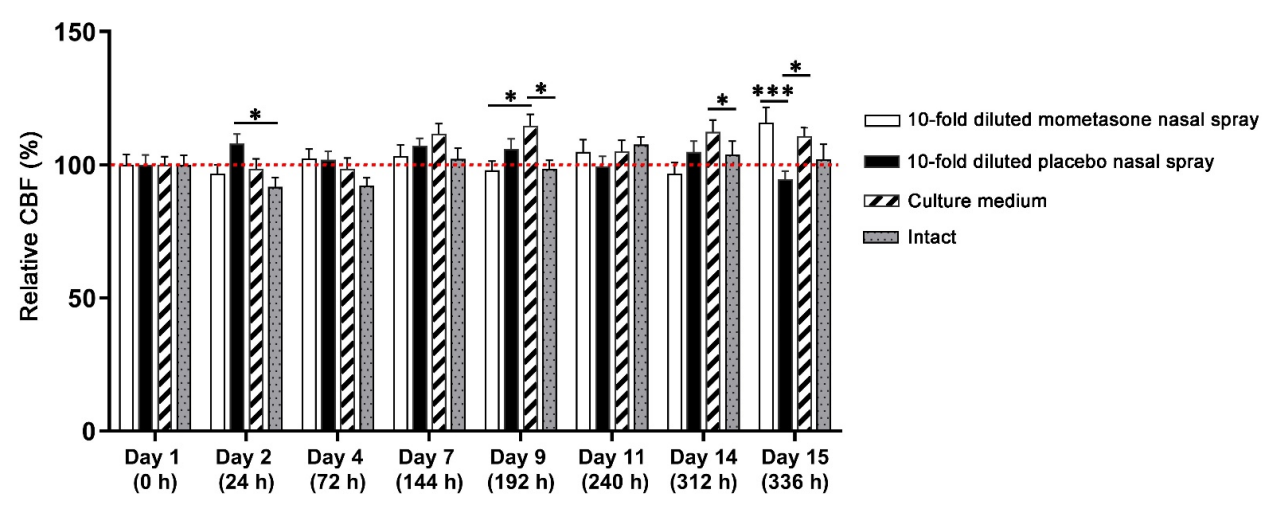

Figure 4. The effect of repeated doses of 10-fold diluted mometasone and placebo nasal spray, culture medium or intact condition on the ciliary beat frequency (CBF) of the nasal MucilAir ${ }^{\mathrm{TM}}$ in vitro model. Results are presented as relative CBF values, expressed as a percentage of the CBF values relative to the corresponding baseline control condition for each tested group measured before treatments. The red dotted line represents $100 \%$ (the value of the control condition before treatment). Each bar represents average relative $\mathrm{CBF} \pm \mathrm{S}$.E. Diluted mometasone and placebo nasal spray do not inhibit $\mathrm{CBF}$ compared to the culture medium-treated and intact nasal in vitro models. Culture medium treatment increases CBF in the second week of daily treatments. $n=50$, two-way ANOVA with Tukey's multiple comparisons test; ${ }^{*} p<0.05,{ }^{* * *} p<0.001$.

\subsection{LDH Cytotoxicity Analysis}

The nasal MucilAir ${ }^{\mathrm{TM}}$ in vitro models were repeatedly exposed for 2 weeks to 10 -fold diluted mometasone nasal spray, 10-fold diluted placebo nasal spray, or culture medium (controls) (Figure 6). Results were compared to the intact nasal MucilAir ${ }^{\mathrm{TM}}$ in vitro models (untreated cultures), which were analysed following the same protocol as the treated cultures. Cytotoxicity was very low in all nasal in vitro models (treated and untreated). Furthermore, 10-fold diluted mometasone or placebo nasal spray did not exhibit significantly different cytotoxicity compared to the controls (culture media-treated and intact cultures) (Figure 6, Supplementary Table S3). These results imply that the tested nasal sprays do not exert higher cytotoxicity than the culture media. 

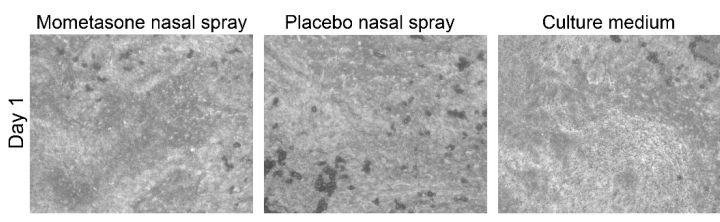
Intact
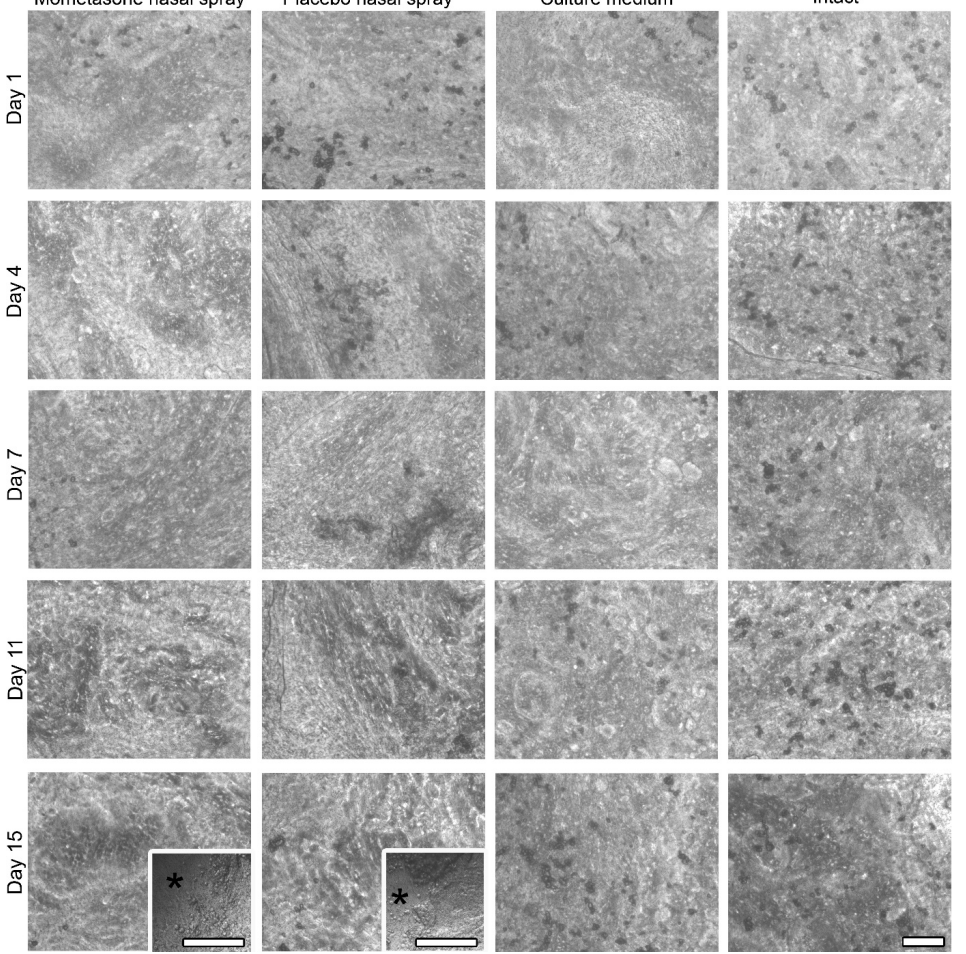

Figure 5. Morphological assessment of the nasal MucilAir ${ }^{\mathrm{TM}}$ in vitro models treated with 10-fold diluted mometasone or placebo nasal spray, culture medium treated or untreated for 14 consecutive days using phase-contrast microscopy. The apical surface view is shown. Cell shedding is observed only at the periphery in the mometasone- and placebo-treated nasal MucilAir ${ }^{\mathrm{TM}}$ in vitro models on the last 2 days of the experiment (inserts, day 15). Asterisks indicate areas without cells. Scale bars, $100 \mu \mathrm{m}$.

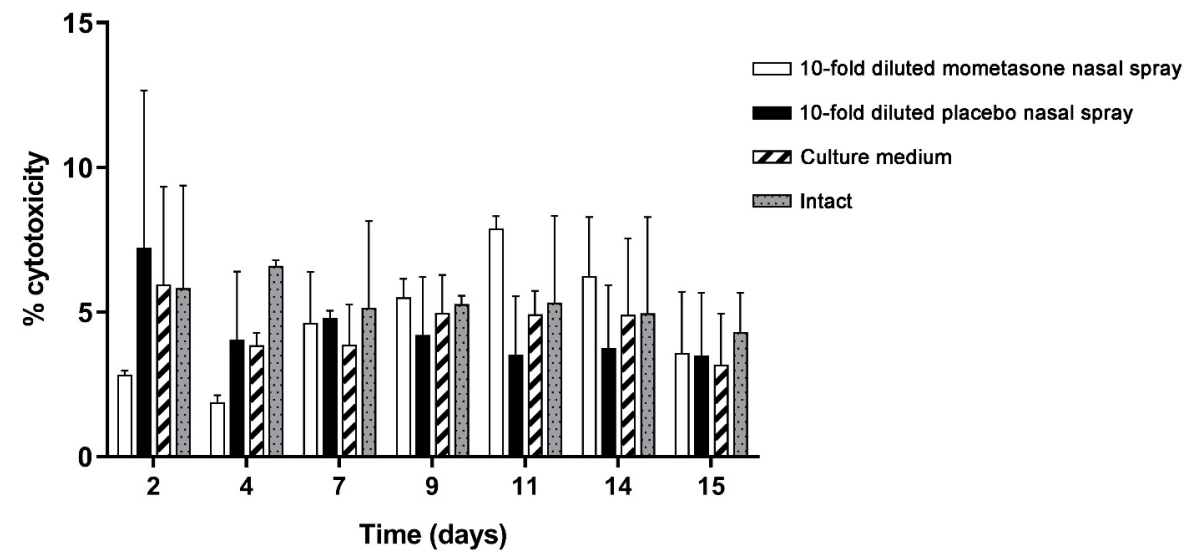

Figure 6. Lactate dehydrogenase (LDH) cytotoxicity assay of repeated doses of 10-fold diluted mometasone and placebo nasal spray, culture medium or intact condition on the nasal MucilAir ${ }^{\mathrm{TM}}$ in vitro model. The results are expressed as a percentage of cytotoxicity relative to the maximum LDH activity (of RPMI 2650 cells). Bars represent mean \pm S.E. of two independent experiments. Two-way ANOVA with Tukey's multiple comparisons test was performed. No statistical differences in cytotoxicity between the treated and untreated nasal in vitro models were observed.

\subsection{Post-Treatment Ultrastructural Analysis}

After 14 days of continuous daily treatment, the diluted mometasone- and placebotreated nasal MucilAir ${ }^{\mathrm{TM}}$ in vitro models were analysed to examine potential nasal spray cytotoxicity. SEM (Figure 7) and TEM (Figure 8) analyses were performed. No significant 
differences in morphology, ultrastructure, or integrity were found between the control and nasal spray-treated nasal in vitro models. SEM analysis showed a similar number of ciliated cells with long cilia in both control (culture media-treated and intact cultures) and nasal spray-treated cultures (mometasone and placebo nasal spray, Figure 7). Furthermore, TEM analysis confirmed tissue integrity in all nasal models, i.e., most epithelial cells were tightly connected via tight junctions, regardless of the treatment (Figure 8). Occasionally, however, disrupted tight junctions were observed in both nasal sprays (mometasone and placebo)-treated and control nasal in vitro models (culture media-treated and untreated cultures). Cell shedding was also observed in control cultures but was less pronounced than in the mometasone- and placebo-treated nasal in vitro models (pronounced in the periphery, Figure 5, Supplementary Figure S2).
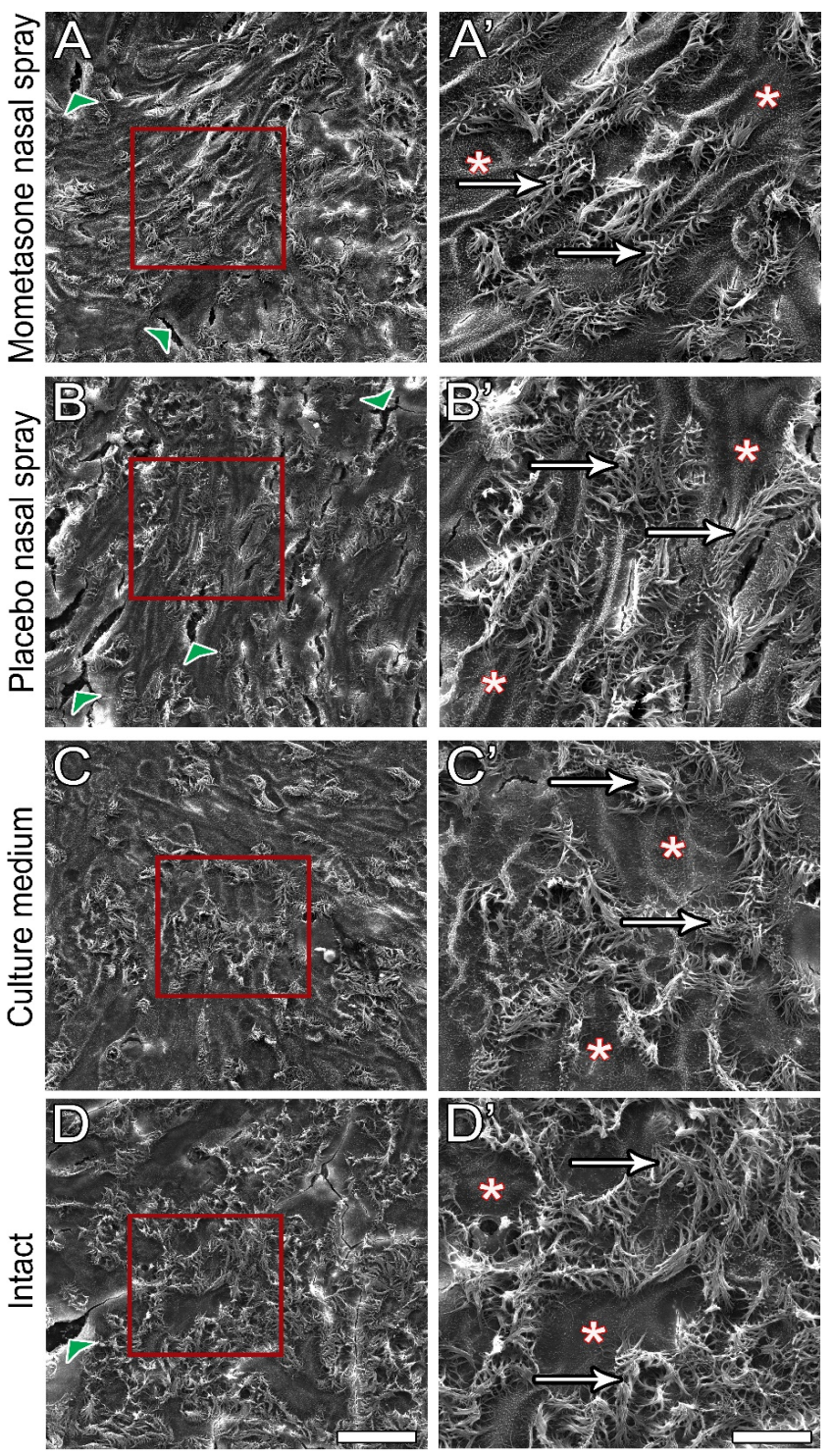

Figure 7. Post-treatment ultrastructural SEM analysis of the apical surface topography of the nasal MucilAir $^{\mathrm{TM}}$ in vitro models. Cultures were treated with 10-fold diluted mometasone nasal spray $\left(\mathbf{A}, \mathbf{A}^{\prime}\right), 10$-fold diluted placebo nasal spray $\left(\mathbf{B}, \mathbf{B}^{\prime}\right)$, or culture medium $\left(\mathbf{C}, \mathbf{C}^{\prime}\right)$ for 14 consecutive days. Intact cultures were not treated $\left(\mathbf{D}, \mathbf{D}^{\prime}\right)$. Ciliated cells (arrows) and non-ciliated cells (asterisks) with apparent cell boundaries are observed in all cultures, regardless of the treatment. Arrowheads indicate artefacts. The red framed areas in images $(\mathbf{A}-\mathbf{D})$ are magnified in $\left(\mathbf{A}^{\prime}-\mathbf{D}^{\prime}\right)$. Scale bars, $50 \mu \mathrm{m}$ (A-D) and $25 \mu \mathrm{m}\left(\mathbf{A}^{\prime}-\mathbf{D}^{\prime}\right)$. 

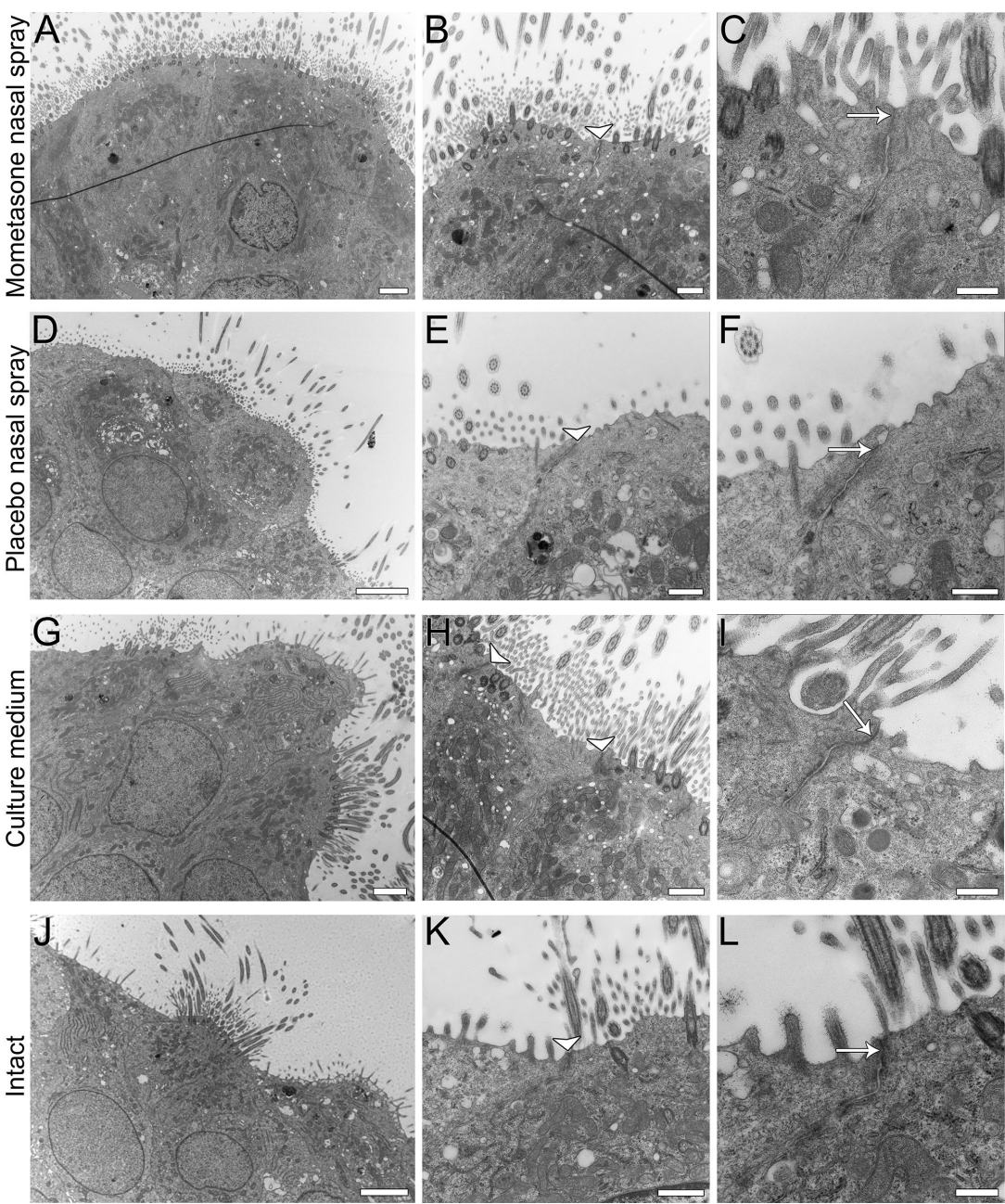

Figure 8. Post-treatment ultrastructural TEM analysis of the nasal MucilAir ${ }^{\mathrm{TM}}$ in vitro models. Cultures were treated with 10-fold diluted mometasone nasal spray (A-C), 10-fold diluted placebo nasal spray (D-F), or culture medium (G-I) for 14 consecutive days. Intact cultures were not treated (J-L). Ciliated and non-ciliated cells are connected with junctional complexes (arrowheads in B,E,H,K) in all MucilAir ${ }^{\mathrm{TM}}$ cultures (treated and untreated). Well-formed tight junctions are seen in higher magnifications (arrows in C,F,I,L). Scale bars, $6 \mu \mathrm{m}(\mathbf{D}), 4 \mu \mathrm{m}(\mathbf{J}), 2 \mu \mathrm{m}(\mathbf{A}, \mathbf{G}), 1 \mu \mathrm{m}(\mathbf{B}, \mathbf{E}, \mathbf{H}, \mathbf{K})$, $600 \mathrm{~nm}(\mathbf{F})$, and $400 \mathrm{~nm}(\mathbf{C}, \mathbf{I}, \mathbf{L})$.

\section{Discussion}

Intranasal administration is a logical delivery choice for the topical treatment of local diseases of the nose and paranasal sinuses, such as allergic and non-allergic rhinitis and sinusitis. Nevertheless, the main purpose of nasal airways is to protect the sensitive lungs from dangerous exposures, and not to serve as an administration route for drugs and vaccines $[2,47]$. It is therefore essential that intranasal formulations are tested for their effects on the integrity of the primary innate nasal defence mechanism, i.e., mucociliary clearance, before use. The aim of this study was to assess the usefulness of the previously developed set-up for ciliotoxicity testing [34] to evaluate the cumulative effect of nasal sprays on ciliary activity in vitro after repeated exposure, as repeated doses of topical nasal formulations are usually used.

4.1. Applicability of the Nasal MucilAir ${ }^{\mathrm{TM}}$ In Vitro Model for the Analysis of Repeated Doses Ciliotoxicity and Cytotoxicity of Topical Nasal Formulations

The nasal MucilAir ${ }^{\mathrm{TM}}$ in vitro model is a fully differentiated pseudostratified epithelium cultured at the air-liquid interface that contains all the structural characteristics of 
nasal (respiratory) mucosa in vivo. Namely, the nasal in vitro models consist of basal, goblet, and ciliated cells connected by tight and adherent junctions (Figures 1, 7 and 8), which are expressed homogenously over the entire apical surface and enable the nasal in vitro model to form a polarized barrier [24]. The nasal MucilAir ${ }^{\mathrm{TM}}$ in vitro model display functional cilia (Video S1), mucus production, ion channel function, and secretion of cytokines, chemokines, and various proteases $[48,49]$. A crucial characteristic of the nasal MucilAir ${ }^{\mathrm{TM}}$ in vitro model is its usefulness for long-term chronic studies and repeated exposure, as it retains functional properties with ciliary beating over several months. Furthermore, cell growth on porous membranes allows morphological and ultrastructural analyses after treatment ([34], http:/ / www.epithelix.com, accessed on 25 August 2021). The nasal MucilAir ${ }^{\mathrm{TM}}$ in vitro model has been used to study repeated exposure to smoke [50-52], gasoline engine emissions [53], formaldehyde [54], a novel hypertonic seawater solution for nasal lavage [55], and inhaled drugs or drug candidates [56]. To the best of our knowledge, our study is the first to investigate the relevance, value, and possible application of the nasal MucilAir ${ }^{\mathrm{TM}}$ in vitro model to predict chronic in vivo ciliotoxicity and cytotoxicity of topical nasal formulations. In addition, this is the first study to examine chronic nasal spray ciliotoxicity in human nasal epithelia in vitro.

\subsection{The Importance of In Vitro Assessment of the Effect of Chronic Topical Nasal Formulation Exposure on $C B F$}

Topical nasal drugs, such as antihistamines, corticosteroids, and decongestants, are commonly used to treat rhinitis, sinusitis, and related allergic or chronic conditions. This is due to their efficacy, tolerability, easy application, cost-effectiveness, and low risk of systemic side effects [1-6]. As topical nasal formulations are often repeatedly applied as long-term therapy, it is important to elucidate their chronic effects on mucociliary clearance and nasal mucosa. Ciliary beating is a driving force for mucociliary clearance. CBF is linearly correlated with mucociliary clearance and can thus be used to quantitatively determine mucociliary function [9]. Defective mucociliary clearance lengthen the contact times of the airways with pathogens, irritating or carcinogenic substances, which can results in respiratory infections and/or damaged mucosa [57]. Some studies have indicated that mucociliary transport or $\mathrm{CBF}$ are decreased in patients with chronic rhinosinusitis, allergic rhinitis, or sinusitis [58,59]. It is therefore of great importance to determine whether topical nasal drugs can hinder nasal ciliary activity.

The in vitro safety assessment of topically administered drugs based on CBF measurements has two important advantages. First, only in vitro experiments guarantee constant conditions and exclude factors that can influence CBF, such as stress, hormone secretion, inflammatory mediators, infections, osmolarity, $\mathrm{pH}$, and temperature [15,60-62]. Second, the use of human nasal in vitro models overcomes the disadvantages of in vivo and ex vivo animal models with respect to species differences. Furthermore, the use of animals is reduced to a minimum in compliance with the guidelines for replacement, reduction, and refinement (3Rs) in laboratory animal use. By contrast, clinical trials are costly, time-consuming, and often burdensome on patients [27]; thus, they are not appropriate for screening a large number of formulation candidates. Initial in vitro screening assays for topical nasal drug formulation candidates represent a rapid, highly effective, and economical alternative to expensive clinical screenings in animals and humans.

However, in vitro experiments also have their limitations and cannot precisely duplicate in vivo conditions. Topical nasal formulations are distributed and diluted on the surface of nasal mucosa after application in vivo. Due to mucociliary transport, drug formulations can be removed from the natural environment in the nose. In addition, epithelial renewal continues in vivo [38]. Thus, in vitro models can lack a protective mucus layer and cannot entirely mimic the process of mucus removal from the nose, as the common technical set-up of cultured cells on inserts does not currently enable this. The dilution of nasal formulations by the produced mucus that is observed in vivo, however, can be adequately replicated also in vitro. Namely, a 5-10-fold dilution of nasal formulation has been proposed to mimic the in vivo situation and more realistically reflect the ciliotoxic 
effect of nasal formulations [46]. Jiao et al. [46] calculated that nasal products are diluted at least 5-fold after administration into the nose, as the healthy human nose contains approximately $0.4 \mathrm{~mL}$ of mucus, and spray doses are usually in the order of $0.1 \mathrm{~mL}$ in clinical practice. As mucus production can be increased in patients with allergic rhinitis, it is reasonable to test the nasal preparations at even greater dilutions. A 10-fold dilution of nasal spray was therefore analysed in our study in addition to undiluted nasal spray. In this way, a more realistic estimate of the drugs' safety for in vivo use can be made. Substances that do not damage ciliated cells or affect mucociliary clearance in vitro can be considered safe for use in vivo [3].

4.3. The Observed Effects of Repeated Exposure to Clinically Relevant Doses of Mometasone Nasal Spray on CBF Are in Good Agreement with Those of In Vivo Studies

Mometasone furoate is a glucocorticosteroid used in nasal sprays as an effective topical medical treatment for seasonal or perennial allergic rhinitis and nasal polyposis [63]. The topical application of mometasone furoate as a nasal spray prevents high systemic concentrations and does not cause clinically significant adverse effects. A clinical trial on patients with allergic rhinitis showed positive results with significant symptom improvement $[64,65]$. Its clinical efficacy combined with a favourable safety and tolerability profile confers its favourable benefit-risk ratio [66]. Naclerio et al. [67] reported that a 2-week treatment with mometasone nasal spray (Nasonex, containing mometasone furoate and BKC) did not impair mucociliary clearance. Similarly, Pata et al. [68] showed that mometasone furoate nasal spray does not affect mucociliary clearance in patients with perennial allergic rhinitis.

In this study, we have shown that the effect of repeated treatment with mometasone nasal spray Mommox ${ }^{\circledR}$ on CBF in the nasal MucilAir ${ }^{\mathrm{TM}}$ in vitro model is dose- and timedependent (Figures 2-5). Undiluted mometasone nasal spray treatment had a cumulative inhibitory effect on CBF and was cytotoxic, whereas a clinically relevant dose of Mommox ${ }^{\circledR}$ spray showed no ciliotoxicity or cytotoxicity. Furthermore, the results obtained with a clinically relevant dose of Mommox ${ }^{\circledR}$ nasal spray, which contains BKC (Figures 4-8), are consistent with the results of clinical studies on Nasonex mometasone nasal spray, which also contains BKC. Nasonex does not impair mucociliary clearance [67] and does not damage nasal mucosa [69]. Moreover, a clinical study has shown that Mommox ${ }^{\circledR}$ nasal spray is effective and well-tolerated in the treatment of seasonal allergic rhinitis [64]. Furthermore, we have also demonstrated that repeated treatment with placebo nasal spray at an undiluted concentration exerts cilio-inhibiting and cytotoxic effects (Figures 2 and 3). Conversely, at clinically relevant doses, it did not exhibit ciliotoxicity (Figures 4-8). Since placebo spray contains excipients without the active drug, we speculate that mometasone furoate itself has no adverse effects on ciliary activity and is not cytotoxic. By contrast, BKC, a preservative in nasal sprays, has been shown to inhibit ciliary activity and damage nasal epithelial cells in vitro $[34,70,71]$. It is therefore possible that the cumulative cilio-inhibitory and cytotoxic effects of undiluted nasal sprays (Mommox ${ }^{\circledR}$ and placebo) could be due to BKC. However, 10-fold diluted Mommox ${ }^{\circledR}$ and placebo did not affect ciliary function and were not cytotoxic. This is consistent with in vivo studies that showed that the toxic effect of BKC in vivo is inactivated by proteins in nasal secretions [33,72].

Interestingly, chronic treatment with culture media exhibited a cilio-stimulatory effect, possibly due to extensive mucus dilution and rinsing. Moreover, it is possible that culture media exerts similar cilio-stimulatory effects as isotonic saline, Ringer Lactate solution, isotonic seawater, etc., which have been shown to increase mucociliary clearance and CBF. These nasal irrigation solutions contain high levels of minerals and trace elements, such as calcium, potassium, magnesium, and zinc ions, which can assist in epithelial wound repair and ciliary beat regulation [3,73-76].

The existing data in the literature suggest that mucociliary clearance of the entire nasal mucosa occurs in as little as $10 \mathrm{~min}[77,78]$ to $20 \mathrm{~min}$ [79], while $30 \mathrm{~min}$ is considered the cutoff point for distinguishing between normal and impaired nasal mucociliary clearance [79]. In our study, $30 \mathrm{~min}$ of daily exposure to nasal sprays was implemented to more closely 
represent the impaired mucociliary clearance time observed in patients [80]. The results of our study suggest that clinically relevant doses of Mommox ${ }^{\circledR}$ nasal spray can be considered safe, as even prolonged exposure (30 $\mathrm{min}$ versus $10 \mathrm{~min}$ ) did not result in ciliotoxicity.

Taken together, our results demonstrate high sensitivity of the in vitro CBF analysis setup in terms of dosing and exposure (repetitions) to topical nasal formulations. Furthermore, there is a good correlation between clinical in vivo and in vitro studies when nasal formulations are appropriately diluted to mimic in vivo conditions. This suggests the usefulness of the described CBF analysis set-up for safety studies regarding repeated exposure.

However, it is important to emphasize the potential limitations to in vitro chronic exposure studies. Ultrastructural analysis of the control nasal in vitro models (culture media-treated and intact cultures) showed that long-term experiments affect the integrity of the nasal MucilAir ${ }^{\mathrm{TM}}$ cultures. Namely, tight junctions were occasionally disrupted, and cell shedding occurred in certain regions by day 15 . Cell shedding was also observed in the nasal spray-treated cultures by day 15 (Figure 5), and we suspect that further treatment and CBF measurements would lead to more extensive cell shedding. Furthermore, the disrupted tight junctions indicate that further treatment and measurements would also lead to more extensive cell detachment. It is therefore possible that chronic in vitro studies using topical nasal formulations and the nasal MucilAir ${ }^{\mathrm{TM}}$ nasal in vitro models may be limited to a period of 2 weeks. However, it is also possible that the reduced integrity of the nasal in vitro models may be due to aging (cell senescence).

\section{Conclusions}

Topical nasal drug formulations are recognized as a first-line treatment for local diseases of the nose and paranasal sinuses. Available data suggest that some active drugs and excipients of nasal formulations impede ciliary function and mucociliary clearance. It is therefore important to assess the effects of intranasal drugs on mucociliary functions before employing them to treat various nasal diseases. In vitro CBF assessment has been shown to be an accurate and reproducible technique. Topical nasal formulations are usually applied as long-term therapy with repeated doses; therefore, it is important to elucidate their chronic versus acute effect on mucociliary clearance and nasal mucosa. Our current study assessed an in vitro assay with clinically relevant doses of nasal formulations intended for chronic treatment and the 3D nasal MucilAir ${ }^{\mathrm{TM}}$ in vitro model, and our results correlate well with those of in vivo studies. This indicates that such an in vitro assay has great potential for topical nasal drug screening and may contribute to the 3Rs approach to animal testing.

Supplementary Materials: The following are available online at https://www.mdpi.com/article/ 10.3390/pharmaceutics13111750/s1, Figure S1: Morphological assessment of the nasal MucilAirTM in vitro models treated with placebo nasal spray, undiluted mometasone nasal spray, and culture media for 10 consecutive days using stereoscopic microscopy, Scale bar, $100 \mu \mathrm{m}$, Figure S2: Morphological assessment of the nasal MucilAirTM in vitro models treated with 10-fold diluted mometasone and placebo nasal spray and culture media for 14 consecutive days using stereoscopic microscopy, Scale bar, $100 \mu \mathrm{m}$, Table S1: Results of two-way ANOVA analysis with Tukey's multiple comparisons test of repeated exposure to undiluted nasal spray, Table S2: Results of two-way ANOVA analysis with Tukey's multiple comparisons test of repeated exposure to diluted nasal spray, Table S3: Results of two-way ANOVA analysis with Tukey's multiple comparisons test of LDH cytotoxicity, Video S1: Baseline CBF, Video S2: Undiluted Mommox-day 1, Video S3: Undiluted Mommox-day 7, Video S4: Undiluted Mommox — day 9, Video S5: Undiluted Placebo—day 1, Video S6: Undiluted Placebo—day 7, Video S7: Undiluted Placebo—day 11, Video S8: Culture medium—day 1, Video S9: Culture medium —day 11, Video S10: Intact—day 1, Video S11: Intact—day 7, Video S12: Diluted Mommox—day 1, Video S13: Diluted Mommox—day 7, Video S14: Diluted Mommox—day 15, Video S15: Diluted Placebo—day 1, Video S16: Diluted Placebo—day 7, Video S17: Diluted Placeboday 15, Video S18: Culture medium—day 1, Video S19: Culture medium—day 9, Video S20: Culture medium—day 14, Video S21: Intact—day 1, Video S22: Intact—day 7, Video S23: Intact—day 14. 
Author Contributions: Conceptualization, K.K. and M.E.K.; methodology, L.T., N.S., K.K. and M.E.K.; validation, L.T., K.K. and M.E.K.; formal analysis, L.T., N.S.; investigation, L.T., M.E.K.; resources, K.K. and M.E.K.; data curation, L.T.; writing-original draft preparation, L.T.; writingreview and editing, L.T., N.S., K.K. and M.E.K.; visualization, L.T. and M.E.K.; supervision, K.K. and M.E.K.; project administration, K.K. and M.E.K.; funding acquisition, K.K. and M.E.K. All authors have read and agreed to the published version of the manuscript.

Funding: The study was supported by Lek Pharmaceuticals, d.d., Sandoz Development Center Slovenia, the Slovenian Research Agency (Grants No. J7-2594, P3-0108), and the MRIC UL IP-0510 Infrastructure program.

Institutional Review Board Statement: Not applicable.

Informed Consent Statement: Not applicable.

Data Availability Statement: The authors declare that they have no known competing financial interests or personal relationships that could have appeared to influence the work reported in this paper.

Acknowledgments: The authors thank Marko Kreft for methodological help and discussions. The authors thank Eva Lasic for editing a draft of this manuscript. The authors also acknowledge the technical assistance of Sanja Čabraja, Nada Pavlica Dubarič, Linda Štrus, and Sabina Železnik.

Conflicts of Interest: The authors declare no conflict of interest. The funders had no role in the design of the study; in the collection, analyses, or interpretation of data; in the writing of the manuscript, or in the decision to publish the results.

\section{References}

1. Watts, A.M.; Cripps, A.W.; West, N.P.; Cox, A.J. Modulation of Allergic Inflammation in the Nasal Mucosa of Allergic Rhinitis Sufferers with Topical Pharmaceutical Agents. Front. Pharm. 2019, 10, 294. [CrossRef] [PubMed]

2. Bitter, C.; Suter-Zimmermann, K.; Surber, C. Nasal drug delivery in humans. Curr. Probl. Derm. 2011, 40, $20-35$.

3. Jiao, J.; Zhang, L. Influence of Intranasal Drugs on Human Nasal Mucociliary Clearance and Ciliary Beat Frequency. Allergy Asthma Immunol. Res. 2019, 11, 306-319. [CrossRef]

4. Seidman, M.D.; Gurgel, R.K.; Lin, S.Y.; Schwartz, S.R.; Baroody, F.M.; Bonner, J.R.; Dawson, D.E.; Dykewicz, M.S.; Hackell, J.M.; Han, J.K.; et al. Clinical practice guideline: Allergic rhinitis. Otolaryngol. Head Neck Surg. 2015, 152 (Suppl. S1), S1-S43. [CrossRef]

5. Weaver, J.M.J.; Ross-Innes, C.S.; Shannon, N.; Lynch, A.G.; Forshew, T.; Barbera, M.; Murtaza, M.; Ong, C.J.; Lao-Sirieix, P.; Dunning, M.J.; et al. Ordering of mutations in preinvasive disease stages of esophageal carcinogenesis. Nat. Genet. 2014, 46, 837-843. [CrossRef]

6. Rosenfeld, R.M.; Piccirillo, J.F.; Chandrasekhar, S.S.; Brook, I.; Ashok Kumar, K.; Kramper, M.; Orlandi, R.R.; Palmer, J.N.; Patel, Z.M.; Peters, A.; et al. Clinical practice guideline (update): Adult sinusitis. Otolaryngol Head Neck Surg. 2015, 152 (Suppl. S2), S1-S39. [CrossRef]

7. Homer, J.J.; Aggarwal, R.; Cordoza, A. Delivery of topical nasal drugs. Am. J. Drug Deliv. 2003, 1, 125-131. [CrossRef]

8. Kern, R.C.; Decker, J.R. Functional Defense Mechanisms of the Nasal Respiratory Epithelium. In Nasal Physiology and Pathophysiology of Nasal Disorders; Önerci, T.M., Ed.; Springer: Berlin/Heidelberg, Germany, 2013; pp. 27-45.

9. Inoue, D.; Furubayashi, T.; Ogawara, K.; Kimura, T.; Higaki, K.; Shingaki, T.; Kimura, S.; Tanaka, A.; Katsumi, H.; Sakane, T.; et al. In vitro evaluation of the ciliary beat frequency of the rat nasal epithelium using a high-speed digital imaging system. Biol. Pharm. Bull. 2013, 36, 966-973. [CrossRef] [PubMed]

10. Gizurarson, S. The effect of cilia and the mucociliary clearance on successful drug delivery. Biol. Pharm. Bull. 2015, 38, 497-506. [CrossRef] [PubMed]

11. Lale, A.M.; Mason, J.D.; Jones, N.S. Mucociliary transport and its assessment: A review. Clin. Otolaryngol Allied Sci. 1998, 23, 388-396. [CrossRef]

12. Rogers, D.F. Physiology of airway mucus secretion and pathophysiology of hypersecretion. Respir. Care 2007, 52, 1134-1146.

13. Chen, J.; Jing, J.; Su, E.; Badger, C.; Coughlan, C.; Chen, Z.; Wong, B. Measurement of Ciliary Beat Frequency Using Ultra-High. Resolution Optical Coherence Tomography; SPIE: San Francisco, CA, USA, 2016; Volume 9689.

14. Klocker, N.; Verse, T.; Rudolph, P. The protective effect of dexpanthenol in nasal sprays. First results of cytotoxic and ciliary-toxic studies in vitro. Laryngorhinootologie 2003, 82, 177-182.

15. Hofmann, T.; Gugatschga, M.; Koidl, B.; Wolf, G. Influence of preservatives and topical steroids on ciliary beat frequency in vitro. Arch. Otolaryngol. Head Neck Surg. 2004, 130, 440-445. [CrossRef]

16. Merkus, P.; Romeijn, S.G.; Verhoef, J.C.; Merkus, F.W.; Schouwenburg, P.F. Classification of cilio-inhibiting effects of nasal drugs. Laryngoscope 2001, 111, 595-602. [CrossRef]

17. Dimova, S.; Maes, F.; Brewster, M.E.; Jorissen, M.; Noppe, M.; Augustijns, P. High-speed digital imaging method for ciliary beat frequency measurement. J. Pharm Pharm. 2005, 57, 521-526. [CrossRef] 
18. Palmberger, T.F.; Augustijns, P.; Vetter, A.; Bernkop-Schnürch, A. Safety assessment of thiolated polymers: Effect on ciliary beat frequency in human nasal epithelial cells. Drug Dev. Ind. Pharm. 2011, 37, 1455-1462. [CrossRef]

19. Vetter, A.; Augustijns, P.; Bernkop-Schnürch, A. Solubilizing agents in nasal formulations and their effect on ciliary beat frequency. Toxicol. Vitr. 2012, 26, 150-156. [CrossRef] [PubMed]

20. Piqué, N.; De Servi, B. Rhinosectan ${ }^{\circledR}$ spray (containing xyloglucan) on the ciliary function of the nasal respiratory epithelium; results of an in vitro study. Allergy Asthma Clin. Immunol. 2018, 14, 41. [CrossRef]

21. Kreft, M.E.; Jerman, U.D.; Lasič, E.; Lanišnik Rižner, T.; Hevir-Kene, N.; Peternel, L.; Kristan, K. The characterization of the human nasal epithelial cell line RPMI 2650 under different culture conditions and their optimization for an appropriate in vitro nasal model. Pharm. Res. 2015, 32, 665-679. [CrossRef] [PubMed]

22. Kreft, M.E.; Tratnjek, L.; Lasič, E.; Hevir, N.; Rižner, T.L.; Kristan, K. Different Culture Conditions Affect Drug Transporter Gene Expression, Ultrastructure, and Permeability of Primary Human Nasal Epithelial Cells. Pharm. Res. 2020, $37,170$. [CrossRef] [PubMed]

23. Huang, S.; Wiszniewski, L.; Constant, S. The Use of In Vitro 3D Cell Models in Drug Development for Respiratory Diseases. In Drug Discovery and Development_-Present and Future; Izet, K., Ed.; InTech: London, UK, 2011.

24. Mercier, C.; Jacqueroux, E.; He, Z.; Hodin, S.; Constant, S.; Perek, N.; Boudard, D.; Delavenne, X. Pharmacological characterization of the 3D MucilAir ${ }^{\mathrm{TM}}$ nasal model. Eur J. Pharm Biopharm 2019, 139, 186-196. [CrossRef]

25. Ehrhardt, C.; Kim, K.-J. Drug Absorption Studies: In Situ, in Vitro and in Silico Models; Springer: Berlin/Heidelberg, Germany, 2007.

26. Salade, L.; Wauthoz, N.; Goole, J.; Amighi, K. How to characterize a nasal product. The state of the art of in vitro and ex vivo specific methods. Int. J. Pharm. 2019, 561, 47-65.

27. Fogel, D.B. Factors associated with clinical trials that fail and opportunities for improving the likelihood of success: A review. Contemp. Clin. Trials Commun. 2018, 11, 156-164. [CrossRef]

28. Klöcker, N.; Rudolph, P.; Verse, T. Evaluation of protective and therapeutic effects of dexpanthenol on nasal decongestants and preservatives: Results of cytotoxic studies in vitro. Am. J. Rhinol. 2004, 18, 315-320. [CrossRef]

29. Verse, T.; Sikora, C.; Rudolph, P.; Klöcker, N. The tolerability of nasal drugs with special regard to preservatives and physicochemical parameters. Laryngorhinootologie 2003, 82, 782-789. [PubMed]

30. Workman, A.D.; Cohen, N.A. The effect of drugs and other compounds on the ciliary beat frequency of human respiratory epithelium. Am. J. Rhinol. Allergy 2014, 28, 454-464. [CrossRef]

31. Hofmann, T.; Wolf, G.; Koidl, B. Effect of topical corticosteroids and topical antihistaminics on ciliary epithelium of human nasal mucosa in vitro. HNO 1998, 46, 146-151. [PubMed]

32. Joki, S.; Saano, V.; Nuutinen, J.; Virta, P.; Karttunen, P.; Silvasti, M.; Toskala, E. Effects of Some Preservative Agents on Rat and Guinea Pig Tracheal and Human Nasal Ciliary Beat Frequency. Am. J. Rhinol. 1996, 10, 181-186. [CrossRef]

33. Riechelmann, H.; Deutschle, T.; Stuhlmiller, A.; Gronau, S.; Bürner, H. Nasal toxicity of benzalkonium chloride. Am. J. Rhinol. 2004, 18, 291-299. [CrossRef] [PubMed]

34. Tratnjek, L.; Kreft, M.; Kristan, K.; Kreft, M.E. Ciliary beat frequency of in vitro human nasal epithelium measured with the simple high-speed microscopy is applicable for safety studies of nasal drug formulations. Toxicol. Vitr. 2020, 66, 104865. [CrossRef] [PubMed]

35. Mallants, R.; Jorissen, M.; Augustijns, P. Beneficial effect of antibiotics on ciliary beat frequency of human nasal epithelial cells exposed to bacterial toxins. J. Pharm. Pharm. 2008, 60, 437-443. [CrossRef]

36. Berg, O.H.; Lie, K.; Steinsvåg, S.K. The effects of topical nasal steroids on rat respiratory mucosa in vivo, with special reference to benzalkonium chloride. Allergy 1997, 52, 627-632. [CrossRef] [PubMed]

37. Cho, J.H.; Kwun, Y.S.; Jang, H.S.; Kang, J.M.; Won, Y.S.; Yoon, H.R. Long-term use of preservatives on rat nasal respiratory mucosa: Effects of benzalkonium chloride and potassium sorbate. Laryngoscope 2000, 110, 312-317. [CrossRef] [PubMed]

38. Lebe, E.; Baka, M.; Yavaşoğlu, A.; Aktuğ, H.; Ateş, U.; Uyanikgil, Y. Effects of preservatives in nasal formulations on the mucosal integrity: An electron microscopic study. Pharmacology 2004, 72, 113-120. [CrossRef] [PubMed]

39. Cüreoğlu, S.; Akkuş, M.; Osma, Ü.; Yaldiz, M.; Oktay, F.; Can, B.; Güven, C.; Tekın, M.; Merıç, F. The effect of benzalkonium chloride on rabbit nasal mucosa in vivo: An electron microscopy study. Eur. Arch. Otorhinolaryngol. 2002, 259, 362-364. [CrossRef] [PubMed]

40. Kuboyama, Y.; Suzuki, K.; Hara, T. Nasal lesions induced by intranasal administration of benzaikonium chloride in rats. J. Toxicol. Sci. 1997, 22, 153-160. [CrossRef] [PubMed]

41. Graf, P. Adverse effects of benzalkonium chloride on the nasal mucosa: Allergic rhinitis and rhinitis medicamentosa. Clin. Ther. 1999, 21, 1749-1755. [CrossRef]

42. Bernstein, I.L. Is the use of benzalkonium chloride as a preservative for nasal formulations a safety concern? A cautionary note based on compromised mucociliary transport. J. Allergy Clin. Immunol. 2000, 105, 39-44. [CrossRef]

43. Mösges, R.; Shah-Hosseini, K.; Hucke, H.P.; Joisten, M.J. Dexpanthenol: An Overview of its Contribution to Symptom Relief in Acute Rhinitis Treated with Decongestant Nasal Sprays. Adv. Ther. 2017, 34, 1850-1858. [CrossRef]

44. Rueden, C.T.; Schindelin, J.; Hiner, M.C.; DeZonia, B.E.; Walter, A.E.; Arena, E.T.; Eliceiri, K.W. ImageJ2: ImageJ for the next generation of scientific image data. BMC Bioinform. 2017, 18, 529. [CrossRef]

45. Schindelin, J.; Arganda-Carreras, I.; Frise, E.; Kaynig, V.; Longair, M.; Pietzsch, T.; Preibisch, S.; Rueden, C.; Saalfeld, S.; Schmid, B.; et al. Fiji: An open-source platform for biological-image analysis. Nat. Methods 2012, 9, 676-682. [CrossRef] [PubMed] 
46. Jiao, J.; Meng, N.; Zhang, L. The effect of topical corticosteroids, topical antihistamines, and preservatives on human ciliary beat frequency. ORL J. Otorhinolaryngol. Relat. Spec. 2014, 76, 127-136. [CrossRef]

47. Djupesland, P.G. Nasal drug delivery devices: Characteristics and performance in a clinical perspective-a review. Drug Deliv. Transl. Res. 2013, 3, 42-62. [CrossRef]

48. Iskandar, A.R.; Mathis, C.; Martin, F.; Leroy, P.; Sewer, A.; Majeed, S.; Kuehn, D.; Trivedi, K.; Grandolfo, D.; Cabanski, M.; et al. 3-D nasal cultures: Systems toxicological assessment of a candidate modified-risk tobacco product. ALTEX 2017, 34, 23-48. [CrossRef]

49. Huang, S.; Wiszniewski, L.; Constant, S.; Roggen, E. Potential of in vitro reconstituted 3D human airway epithelia (MucilAir ${ }^{\mathrm{TM}}$ ) to assess respiratory sensitizers. Toxicol. Vitr. 2013, 27, 1151-1156. [CrossRef] [PubMed]

50. Iskandar, A.R.; Martin, F.; Talikka, M.; Schlage, W.K.; Kostadinova, R.; Mathis, C.; Hoeng, J.; Peitsch, M.C. Systems approaches evaluating the perturbation of xenobiotic metabolism in response to cigarette smoke exposure in nasal and bronchial tissues. Biomed. Res. Int. 2013, 2013, 512086. [CrossRef]

51. Talikka, M.; Kostadinova, R.; Xiang, Y.; Mathis, C.; Sewer, A.; Majeed, S.; Kuehn, D.; Frentzel, S.; Merg, C.; Geertz, M.; et al. The response of human nasal and bronchial organotypic tissue cultures to repeated whole cigarette smoke exposure. Int. J. Toxicol. 2014, 33, 506-517. [CrossRef] [PubMed]

52. Czekala, L.; Wieczorek, R.; Simms, L.; Yu, F.; Budde, J.; Trelles Sticken, E.; Rudd, K.; Verron, T.; Brinster, O.; Stevenson, M.; et al. Multi-endpoint analysis of human 3D airway epithelium following repeated exposure to whole electronic vapor product aerosol or cigarette smoke. Curr. Res. Toxicol. 2021, 2, 99-115. [CrossRef] [PubMed]

53. Rossner, P., Jr.; Cervena, T.; Vojtisek-Lom, M.; Vrbova, K.; Ambroz, A.; Novakova, Z.; Elzeinova, F.; Margaryan, H.; Beranek, V.; Pechout, M.; et al. The Biological Effects of Complete Gasoline Engine Emissions Exposure in a 3D Human Airway Model (MucilAir(TM)) and in Human Bronchial Epithelial Cells (BEAS-2B). Int. J. Mol. Sci. 2019, 20, 5710. [CrossRef] [PubMed]

54. Constant, S.; Wiszniewski, L.; Huang, S. The use of in vitro 3D cell model of human airway epithelia (MucilAir ${ }^{\mathrm{TM}}$ ) in inhalation toxicity. In Cellular In Vitro Testing: Methods and Protocols; Haycock, J., Ahluwalia, A., Malcol, J., Eds.; CRC Press: Boca Raton, FL, USA, 2014.

55. Huang, S.; Constant, S.; De Servi, B.; Meloni, M.; Culig, J.; Bertini, M.; Saaid, A. In vitro safety and performance evaluation of a seawater solution enriched with copper, hyaluronic acid, and eucalyptus for nasal lavage. Med. Devices 2019, 12, 399-410. [CrossRef] [PubMed]

56. Balogh Sivars, K.; Sivars, U.; Hornberg, E.; Zhang, H.; Brändén, L.; Bonfante, R.; Huang, S.; Constant, S.; Robinson, I.; Betts, C.J.; et al. A 3D Human Airway Model Enables Prediction of Respiratory Toxicity of Inhaled Drugs In Vitro. Toxicol. Sci. 2018, 162, 301-308. [CrossRef]

57. Marttin, E.; Schipper, N.G.M.; Verhoef, J.C.; Merkus, F.W.H.M. Nasal mucociliary clearance as a factor in nasal drug delivery. Adv. Drug Deliv. Rev. 1998, 29, 13-38. [CrossRef]

58. Sun, S.S.; Hsieh, J.F.; Tsai, S.C.; Ho, Y.J.; Kao, C.H. Evaluation of nasal mucociliary clearance function in allergic rhinitis patients with technetium 99m-labeled macroaggregated albumin rhinoscintigraphy. Ann. Otol. Rhinol. Laryngol. 2002, 111, 77-79. [CrossRef]

59. Antunes, M.B.; Gudis, D.A.; Cohen, N.A. Epithelium, cilia, and mucus: Their importance in chronic rhinosinusitis. Immunol. Allergy Clin. North. Am. 2009, 29, 631-643. [CrossRef]

60. Ingels, K.J.; Kortmann, M.J.; Nijziel, M.R.; Graamans, K.; Huizing, E.H. Factors influencing ciliary beat measurements. Rhinology 1991, 29, 17-26. [PubMed]

61. Clary-Meinesz, C.F.; Cosson, J.; Huitorel, P.; Blaive, B. Temperature effect on the ciliary beat frequency of human nasal and tracheal ciliated cells. Biol. Cell 1992, 76, 335-338. [CrossRef]

62. Salathe, M. Regulation of mammalian ciliary beating. Annu. Rev. Physiol. 2007, 69, 401-422. [CrossRef] [PubMed]

63. Small, C.B.; Hernandez, J.; Reyes, A.; Schenkel, E.; Damiano, A.; Stryszak, P.; Staudinger, H.; Danzig, M. Efficacy and safety of mometasone furoate nasal spray in nasal polyposis. J. Allergy Clin. Immunol. 2005, 116, 1275-1281. [CrossRef] [PubMed]

64. Kuna, P.; Wasiak, W.; Jones, S.; Kreft, K.Z. Comparative safety and efficacy of two formulations of mometasone nasal spray in adult seasonal allergic rhinitis. Allergy Asthma Proc. 2014, 35, 332-337. [CrossRef] [PubMed]

65. Meltzer, E.O.; Jalowayski, A.A.; Orgel, H.A.; Harris, A.G. Subjective and objective assessments in patients with seasonal allergic rhinitis: Effects of therapy with mometasone furoate nasal spray. J. Allergy Clin. Immunol 1998, 102, 39-49. [CrossRef]

66. Zitt, M.; Kosoglou, T.; Hubbell, J. Mometasone furoate nasal spray: A review of safety and systemic effects. Drug Saf. 2007, 30, 317-326. [CrossRef] [PubMed]

67. Naclerio, R.M.; Baroody, F.M.; Bidani, N.; De Tineo, M.; Penney, B.C. A comparison of nasal clearance after treatment of perennial allergic rhinitis with budesonide and mometasone. Otolaryngol. Head Neck Surg. 2003, 128, 220-227. [CrossRef] [PubMed]

68. Pata, Y.S.; Akbaş, Y.; Unal, M.; Görür, K.; Ozcan, C.; Vayisoğlu, Y. The effect of mometasone furoate on mucociliary clearance in patients with perennial allergic rhinitis. Kulak Burun Bogaz Ihtis. Derg. 2003, 11, 97-99. [PubMed]

69. Minshall, E.; Ghaffar, O.; Cameron, L.; O’Brien, F.; Quinn, H.; Rowe-Jones, J.; Davies, R.J.; Prior, A.; Lund, V.J.; Mackay, I.S.; et al. Assessment by nasal biopsy of long-term use of mometasone furoate aqueous nasal spray (Nasonex) in the treatment of perennial rhinitis. Otolaryngol. Head Neck Surg. 1998, 118, 648-654.

70. Ho, C.Y.; Wu, M.C.; Lan, M.Y.; Tan, C.T.; Yang, A.H. In vitro effects of preservatives in nasal sprays on human nasal epithelial cells. Am. J. Rhinol. 2008, 22, 125-129. [CrossRef] 
71. Berg, O.H.; Henriksen, R.N.; Steinsvåg, S.K. The effect of a benzalkonium chloride-containing nasal spray on human respiratory mucosa in vitro as a function of concentration and time of action. Pharm. Toxicol. 1995, 76, 245-249. [CrossRef]

72. Richards, D.H. Preservation of nasal sprays. J. Allergy Clin. Immunol. 2000, 106, 595-596. [CrossRef]

73. Hauptman, G.; Ryan, M.W. The effect of saline solutions on nasal patency and mucociliary clearance in rhinosinusitis patients. Otolaryngol. Head Neck Surg. 2007, 137, 815-821. [CrossRef]

74. Ural, A.; Oktemer, T.K.; Kizil, Y.; Ileri, F.; Uslu, S. Impact of isotonic and hypertonic saline solutions on mucociliary activity in various nasal pathologies: Clinical study. J. Laryngol. Otol. 2009, 123, 517-521. [CrossRef]

75. Unal, M.; Görür, K.; Ozcan, C. Ringer-Lactate solution versus isotonic saline solution on mucociliary function after nasal septal surgery. J. Laryngol. Otol. 2001, 115, 796-797. [CrossRef]

76. Bonnomet, A.; Luczka, E.; Coraux, C.; de Gabory, L. Non-diluted seawater enhances nasal ciliary beat frequency and wound repair speed compared to diluted seawater and normal saline. Int. Forum. Allergy Rhinol. 2016, 6, 1062-1068. [CrossRef] [PubMed]

77. Paul, P.; Johnson, P.; Ramaswamy, P.; Ramadoss, S.; Geetha, B.; Subhashini, A. The effect of ageing on nasal mucociliary clearance in women: A pilot study. Int. Sch. Res. Not. 2013, 2013, 1-5. [CrossRef]

78. Koparal, M.; Kurt, E.; Altuntas, E.E.; Dogan, F. Assessment of mucociliary clearance as an indicator of nasal function in patients with COVID-19: A cross-sectional study. Eur. Arch. Otorhinolaryngol. 2021, 278, 1863-1868. [CrossRef]

79. Deborah, S.; Prathibha, K. Measurement of nasal mucociliary clearance. Clin. Res. Pulmonol. 2014, 2, 1019.

80. Sakakura, Y.; Majima, Y.; Harada, T.; Hattori, M.; Ukai, K. Nasal Mucociliary Transport of Chronic Sinusitis in Children. Arch. Otolaryngol. Head Neck Surg. 1992, 118, 1234-1237. [CrossRef] [PubMed] 